\title{
GRHL2 Upregulation Predicts a Poor Prognosis and Promotes the Resistance of Serous Ovarian Cancer to Cisplatin
}

This article was published in the following Dove Press journal: OncoTargets and Therapy

\author{
Yanting Nie \\ Yiling Ding \\ Mengyuan Yang \\ Department of Obstetrics and \\ Gynecology, Second Xiangya Hospital of \\ Central South University, Changsha, \\ People's Republic of China
}

Background: GRHL2 has been shown to function in ovarian carcinogenesis. However, the relationship between GRHL2 and cisplatin (DDP) resistance in serous ovarian cancer (SOC) is not clear. The purpose of this study was to elucidate the function and mechanism of GRHL2 in DDP resistance of SOC.

Materials and Methods: Immunohistochemistry (IHC) was utilized to identify GRHL2 protein expression in DDP resistant and sensitive SOC tissues. GRHL2 mRNA and protein levels were identified using quantitative real-time PCR (qRT-PCR) and Western blotting in SKOV3/DDP and SKOV3 cell lines. We conducted loss- and gain-of-function experiments to uncover the consequence of GRHL2 knockdown or overexpression on the sensitivity of ovarian cancer cells to DDP in vitro and in vivo and the underlying mechanism.

Results: It was observed that expression of GRHL2 was higher in DDP resistant SOC tissues relative to DDP sensitive SOC tissues. In addition, the increased expression of GRHL2 led to shorter progression-free survival (PFS) and overall survival (OS). Meanwhile, the GRHL2 transcript and protein levels in SKOV3/DDP were also higher than SKOV3. Small hairpin RNA (shRNA)-facilitated GRHL2 gene knockdown considerably heightened the sensitivity of SKOV3/DDP cells to DDP by inhibiting proliferation and promoting apoptosis, while upregulation of GRHL2 significantly reduced the sensitivity of SKOV3 cells to DDP by promoting proliferation and decreasing apoptosis. In addition, GRHL2 promotes DDP resistance of SOC through activation of ERK/MAPK signaling pathways.

Conclusion: Our results suggest that GRHL2 up-regulation predicts a poor prognosis and promotes the resistance of SOC to DDP. Therefore, GRHL2 may be a possible treatment target for cisplatin-resistant serous ovarian cancer.

Keywords: serous ovarian cancer, SOC, GRHL2, cisplatin resistance, ERK/MAPK

\section{Introduction}

Serous ovarian cancer, a majority histological type of epithelial ovarian cancer, is a common malignant cancer affecting the female reproductive system. ${ }^{1-3}$ At present, platinum-based combined chemotherapy has been considered to be a classical and effective treatment for SOC. ${ }^{4}$ Despite the high rate of sensitivity to initial chemotherapy in patients with SOC, most patients die of recurrence and metastasis due to drug resistance, with the 5-year survival being only $30-40 \%{ }^{4-6}$ Cisplatin is most commonly used as the front-line chemotherapeutic agent in SOC. ${ }^{7}$ However, cisplatin resistance is the most prevalent clinical problem associated with SOC and the underlying mechanisms remain poorly understood. Therefore, investigating
Correspondence: Mengyuan Yang

Email yangmengyuan_xy@csu.edu.cn 
the mechanism of cisplatin resistance is undoubtedly important and is the basis for exploring novel strategies.

Grainyhead-like 2 (GRHL2), part of the Granular headlike transcription factors family, is not only involved in regulating a series of cellular processes, such as epithelial cell differentiation, epidermis barrier formation, epidermis injury repair, neural tube closure, cholangiocyte maturation, placenta formation, and hearing loss, ${ }^{8-13}$ but also has a vital function in the manifestation and development of breast, ${ }^{14,15}$ colon, ${ }^{16-18}$ gastric, ${ }^{19}$ pancreatic cancer, ${ }^{20}$ as well as oral squamous cell carcinoma and prostate cancer. ${ }^{21-23}$ In addition, GRHL2 plays an important role as a gatekeeper in ovarian cancer EMT through miR-200-ZEB1. ${ }^{24}$ Faddaoui et al demonstrated that GRHL2 encouraged the proliferation, invasion and migration of epithelial ovarian cancer cells. ${ }^{25}$ Conversely, the association between the expression of GRHL2 and the sensitivity of SOC to cisplatin is not clear and needs to be additionally clarified. We conducted this study to help describe the relevance of GRHL2 to the sensitivity of SOC to cisplatin. In this study, we evaluated GRHL2 expression in cisplatin sensitive or resistant SOC tissues and cell lines. Then, we used functional acquisition and loss analysis to examine the function of GRHL2 in the resistance of SOC cells to cisplatin and its potential molecular mechanism.

\section{Materials and Methods}

\section{Patients and Samples}

The study included a total of 80 primary SOC patients that underwent cytoreductive surgery, and received six doses of cisplatin plus paclitaxel adjuvant chemotherapy from January 2012 to Jun 2014 at the Second Xiangya Hospital of Central South University (CSU). The Ethics Committee of Second Xiangya Hospital of CSU granted approval to the study. Patients gave informed consent for utilization of their samples in the study. Clinicopathological characteristics were collected of each patient included age, FIGO stage, histopathological grade, involvement of lymph node, residual tumor magnitude, and recurrence.

\section{Follow-Up}

All patients enrolled in this study were regularly followed-up every 3 months until June 30, 2019 to obtain recurrence and survival information. Follow-up included a complete history of the disease, physical examination, abdominal ultrasonography, blood tests, CT scan of the abdomen and chest to exclude metastasis and recurrence. Recurrence was evaluated as per the guidelines set out by the response evaluation criteria in solid tumors (RECIST) ${ }^{26}$ Development of progressive disease within six months of initial treatment was grouped as chemoresistant, while others were grouped as chemosensitive. ${ }^{27}$ PFS was determined from the time of surgery to disease progression, while OS represents the time duration between cytoreductive surgery and death or, as the case may be, date of latest follow-up.

\section{Immunohistochemistry (IHC)}

The SOC tissues were fixed in 10\% formalin and embedded in paraffin. Sections $(4 \mu \mathrm{m})$ were divided up for IHC studies. Paraffin-embedded tissue specimens were stained for GRHL2 (ab-86,611, Abcam, UK) through the streptavidinperoxidase system (Zhongshan Golden bridge Biotechnology, Beijing, China) as per established guidelines. Only nuclear staining was considered positive for GRHL2. The criteria of IHC results were reported by Shimizu et al. ${ }^{28} \mathrm{~A}$ : according to the cell staining intensity score: 0 : no staining; 1 : light staining; 2: deep staining. B: According to the proportion of cell staining, score of 1 was defined as less than $1 / 3$, score of 2 was defined as $1 / 3$ to $2 / 3$, and score of 3 was defined as more than $2 / 3$. The product of $A$ and $B$ is used as the scoring criterion: 0 is defined as "-"; 1 to 2 is defined as "+"; 3 to 4 is defined as "++", and 5 to 6 is defined as "+++". "." "and" + "are defined as low expression, “++" and "++1" are defined as high expression.

\section{Cell Culture}

Human ovarian serous papillary cystadenocarcinoma cell line (SKOV3) and the SKOV3 cisplatin-tolerant cell line (SKOV3/DDP) were bought from the Cancer Hospital Chinese Academy of Medical Sciences. All cells were maintained in the RPMI 1640 (Hyclone, USA) medium that was supplemented with $10 \%$ fetal bovine serum (FBS), $100 \mathrm{U} / \mathrm{mL}$ penicillin, and $100 \mu \mathrm{g} / \mathrm{mL}$ streptomycin in an incubator at $37^{\circ} \mathrm{C}$ with $5 \% \mathrm{CO} 2$.

\section{Quantitative Real-Time PCR (qRT-PCR)}

Total RNA was isolated through the Trizol reagent (Invitrogen, USA) as per established guidelines. Reverse transcription was conducted using SuperScript First-Strand Synthesis System for RT-PCR (Invitrogen, USA), and qRTPCR was conducted through SYBR Green reporter. Primers had the following sequences: GRHL2 forward, 5'-GAAA ACCGAGTGCAAGTCCTA-3' and reverse, 5'-GGGCCAT GAAAACTGGTGTG-3'; $\beta$-actin forward, 5'- ACCCTG AAGTACCCCATCGAG $-3^{\prime}$ and reverse, 5'- AGCACAGC CTGGATAGCAAC $-3^{\prime}$. $\beta$-actin was used for internal 
controls for GRHL2. $2^{-\Delta \Delta \mathrm{Ct}}$ method was used to analyze the data. Each experiment was established in triplicate.

\section{Vector Construction and Transfection}

The GRHL2 knockdown and overexpression lentiviral vectors were bought from GeneChem (Shanghai, China). Three small hairpin RNAs (shRNA) targeted to different regions of human GRHL2 and a scramble shRNA were linked with lentiviral vector ( $\mathrm{pLV}$-mCherry). The GRHL2 knockdown sequences were: shGRHL2-1, CCAGTGAACCTTTCCCTAAAT; shGRHL2-2, AGACATCAAGTGGCACATTTC; shGRHL2 -3, CGTAGCAATAAACCCATTCAT. Meanwhile, pLVEGFP vector contained the full length of GRHL2 (NM_001330593.1) sequence was transfected in SKOV3 cells. The Empty pLV-EGFP vector was functioned as a negative control.

\section{Western Blot Analysis}

Protein was isolated from cultured cells by lysis through the use of RIPA buffer that contained $0.2 \mathrm{mM}$ PMSF. Protein was run on a SDS-PAGE minigel and transferred to a PVDF membrane. Primary antibodies included anti- $\beta$ actin (60,008-1-Ig), anti-Cyclin D1 (26,939-1-AP), antiFas (60,196-1-Ig), anti-caspase3 (66,470-2-Ig), anti-ERK (16,443-1-AP) and anti-pERK (20,582-1-AP) from Proteintech, USA; anti-GRHL2 (ab-86,611), anti-Ki-67 (ab-16,667) from Abcam, UK. The Bio Rad imaging system (Hercules, USA) was used to detect the band intensities. $\beta$-actin served as an endogenous reference. Experiments were carried out in triplicate.

\section{Cell Proliferation Assays}

Cell proliferation rates were quantified through the use of Cell Counting Kit-8 (CCK-8) (Dojindo, Japan). $1 \times 10^{4}$ cells were added onto each well in a 96-well plate for 24, 48 and 72 hours. CCK-8 reagents $(10 \mu \mathrm{L})$ were supplemented onto each well 4 hours prior to the endpoint of the incubation period. Each well's absorbance value was evaluated using a microplate reader at $450 \mathrm{~nm}$. Experiments were carried out in triplicate.

\section{Determination of Half-Maximal Inhibitory Concentration $\left(\mathrm{IC}_{50}\right)$ Value}

Overall, $1 \times 10^{4}$ cells were added into each well of a 96-well plate and treated using DDP concentrations ranging from $1 \mu \mathrm{g} / \mathrm{mL}$ to $500 \mu \mathrm{g} / \mathrm{mL}$ for 24 hours. The cell viability was measured by $\mathrm{CCK}-8 . \mathrm{IC}_{50}$ value of ovarian cancer cells was calculated by SPSS 23.0 software. Experiments were carried out in triplicate.

\section{Flow Cytometric (FCM) Detection of Apoptosis}

Cell apoptosis was measured by utilizing the Annexin V-allophycocyanin (APC)/Propidium Iodide (PI) Apoptosis Detection Kit (KeyGen, China) as per established guidelines. Experiments were carried out in triplicate. Cell apoptosis rates were determined by FCM.

\section{Chemotherapy Assay in vivo}

The Institutional Animal Care and Use Committee of Second Xiangya Hospital of CSU granted approval to the animal study. The stably transfected cells were amassed and suspended in $100 \mu \mathrm{L}$ PBS at a density of $1 \times 10^{7}$ cells $/ \mathrm{mL}$, and then subcutaneously injected into the left portion of the posterior flank of BALB/c female nude mice (4-6 weeks old, $n=3$ per group). The tumors were examined once every 3 days. The tumor volume was quantified using this equation: Volume $=\left(\right.$ Length $\times$ Width $\left.^{2}\right) / 2\left(\mathrm{~mm}^{3}\right)$. Chemotherapy regimens were initiated once the average tumor size extended to approximately $50 \mathrm{~mm}^{3}$. DDP was administered using intraperitoneal injection at a dose of $3.0 \mathrm{mg} / \mathrm{kg}$ six times with a gap of 3 days. 30 days postsubcutaneous inoculation, mice were sacrificed and the tumors were extracted for further analysis.

\section{Statistical Analysis}

The version 23.0 of SPSS (Chicago, IL, USA) software was utilized for all the statistical analysis. The association between GRHL2 expression and clinicopathological parameters in SOC patients was determined by chi-square test. Continuous variables were presented as mean \pm standard deviation (SD). Independent $t$-tests helped estimate the difference among two groups. Survival curves were analyzed by Kaplan-Meier curves. The Cox proportional hazards regression model was used to recognize factors that were independently related to OS and PFS, and hazard ratio (HR) with 95\% confidence intervals (CI) were obtained. P-value $<0.05$ represents statistical significance.

\section{Results}

\section{High GRHL2 Expression Is Related to Chemotherapy Resistance and Poor Prognosis in SOC}

To investigate the relationship between GRHL2 expression and cisplatin resistance of SOC, immunohistochemistry was 
performed on primary SOC specimens derived from 80 patients. Using the criteria of IHC results reported by Shimizu et al, $^{28}$ the cohort was allotted into GRHL2 high and low expression group. It was observed that GRHL2 was higher expressed in DDP resistant SOC tissues relative to DDP sensitive SOC tissues (Figure 1A), and that the expression score of GRHL2 in resistant SOC tissues $(n=29)$ was drastically higher than those in sensitive SOC tissues $(n=51)$ (Figure 1B). Furthermore, the expression of GRHL2 was correlated to FIGO stage, histological grade and lymph node invasion (Table 1). Further analysis showed that high GRHL2 expression was correlated with short PFS and OS in
A

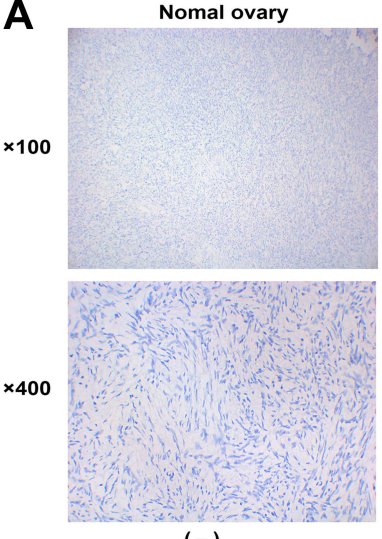

C

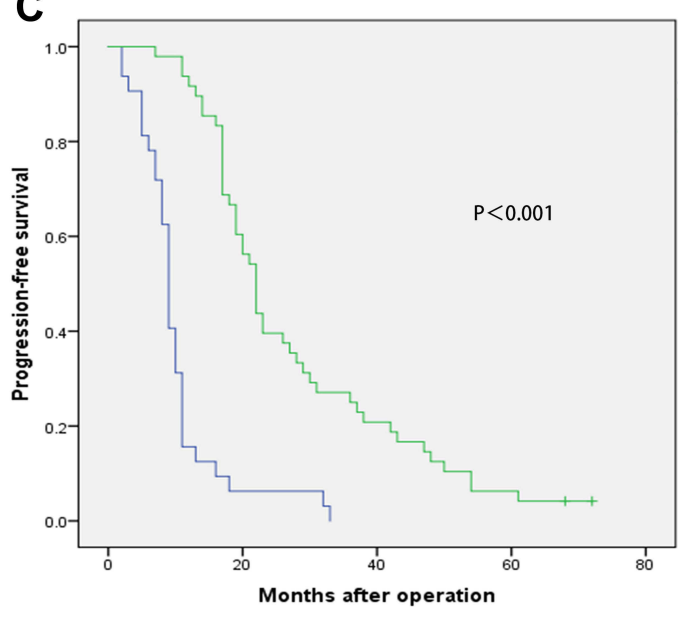

E

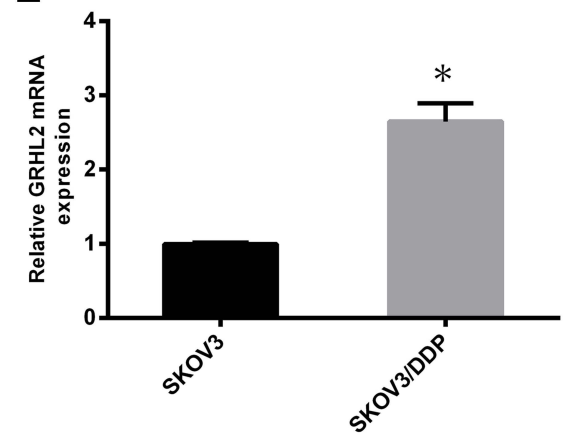

sensitive SOC
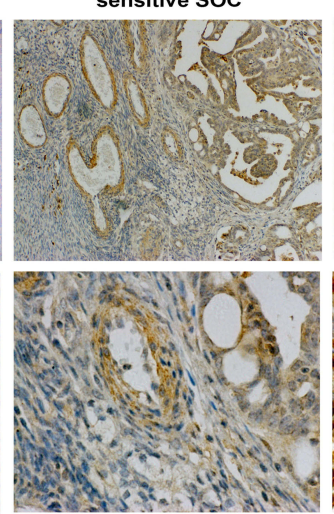

low

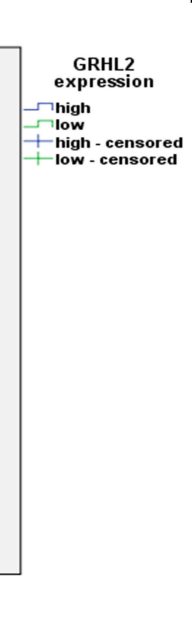

$\mathbf{F}$

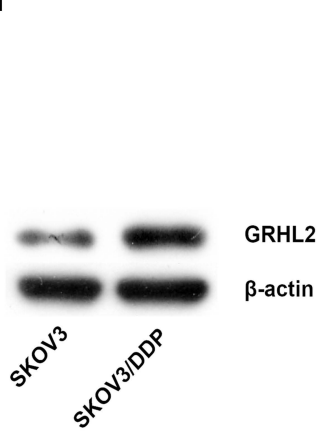

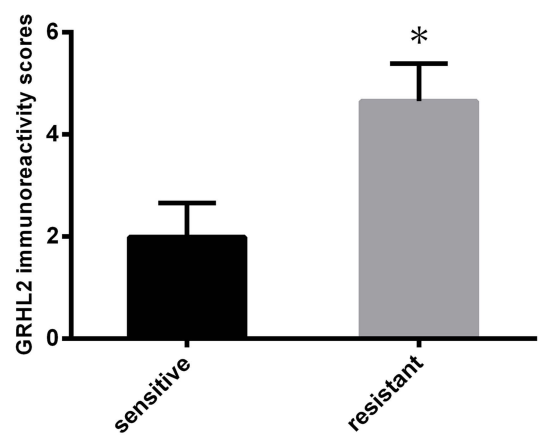

high resistant Soc B

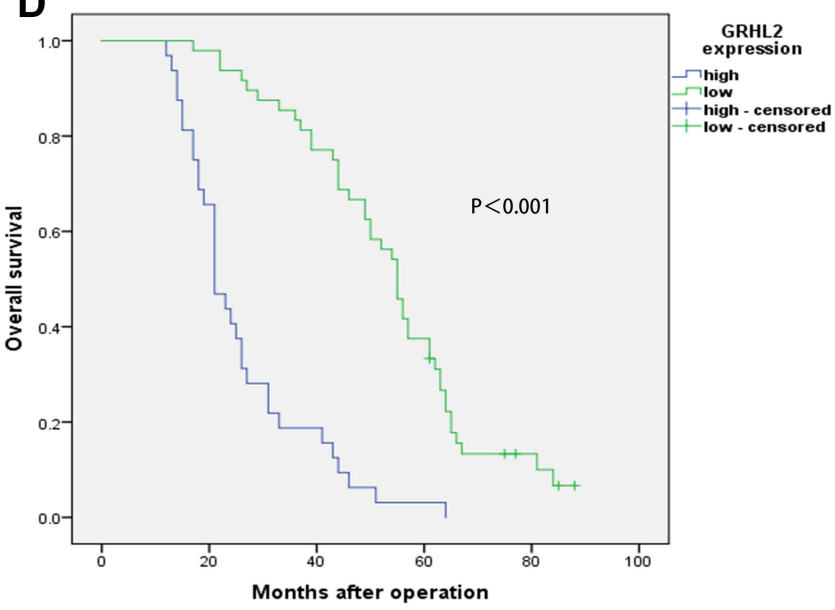

G

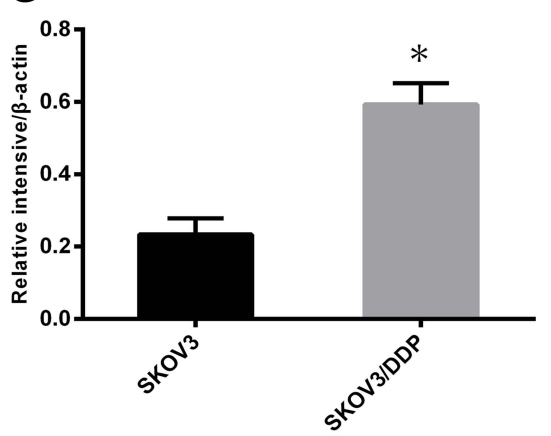

Figure I The expression levels of GRHL2 were up-regulated in the cisplatin-resistant SOC tissues and cells, which affected the prognosis of SOC patients. (A) The expression levels of GRHL2 protein in normal ovary and SOC tissues $(n=80)$ were detected by immunohistochemistry. (magnification, $\times 100, \times 400)(B)$ The expression score of GRHL2 in resistant SOC tissues $(n=29)$ was drastically higher than those in sensitive SOC tissues $(n=51)$. $* P<0.05$ vs sensitive. (C, $\mathbf{D})$ Progression-free survival curves and overall survival curves of SOC patients were analyzed by Kaplan-Meier according to GRHL2 expression ( $n=80)$. (E-G) The relative mRNA and protein levels of GRHL2 were detected in SKOV3/DDP and SKOV3 cells. ${ }^{*} P<0.05$ vs SKOV3.

Note: Data were expressed as mean \pm SD.

Abbreviation: SOC, serous ovarian cancer. 
Table I Association Between GRHL2 Expression and Clinical Characteristics of SOC Patients

\begin{tabular}{|c|c|c|c|c|}
\hline \multirow{2}{*}{ Variables } & \multirow{2}{*}{$\mathbf{N}$} & \multicolumn{2}{|c|}{ GRHL2 Expression } & \multirow{2}{*}{$\begin{array}{l}P \\
\text { value }\end{array}$} \\
\hline & & $\begin{array}{l}\text { Low } \\
\text { Expression }\end{array}$ & $\begin{array}{l}\text { High } \\
\text { Expression }\end{array}$ & \\
\hline Age (year) & & & & 0.690 \\
\hline$<50$ & 35 & 14 & 21 & \\
\hline$\geq 50$ & 45 & 20 & 25 & \\
\hline FIGO stage & & & & $<0.001$ \\
\hline I-II & 18 & 15 & 3 & \\
\hline III-IV & 62 & 19 & 43 & \\
\hline Histological grade & & & & $<0.001$ \\
\hline Low & 21 & 18 & 3 & \\
\hline High & 59 & 16 & 43 & \\
\hline Lymph node & & & & $<0.001$ \\
\hline invasion & & & & \\
\hline Negative & 48 & 32 & 16 & \\
\hline Positive & 32 & 2 & 30 & \\
\hline Tumor residual $(\mathrm{cm})$ & & & & 0.476 \\
\hline Optimal $(\leq 1)$ & 41 & 19 & 22 & \\
\hline Sub-optimal $(>1)$ & 39 & 15 & 24 & \\
\hline Chemotherapeutic & & & & $<0.001$ \\
\hline response & & & & \\
\hline Sensitive & 51 & 32 & 19 & \\
\hline Resistant & 29 & 2 & 27 & \\
\hline
\end{tabular}

Note: The bold number represents the P-values with significant differences $(\mathrm{P}<0.05)$.

Abbreviation: $\mathrm{N}$, sample number.

SOC patients (Figure 1C and D). Moreover, univariate and multivariate analyses revealed late FIGO stage and high GRHL2 expression were independent risk factor associated with recurrence and poor prognosis in SOC patients (Tables 2 and 3). Therefore, our results suggested that GRHL2 played a role in cisplatin resistance of SOC, which affected the prognosis of SOC patients.

\section{High GRHL2 mRNA and Protein} Expression in Cisplatin-Resistant Ovarian Serous Papillary Cystadenocarcinoma

\section{Cell Line (SKOV3/DDP)}

We performed qRT-PCR and Western blot to identify GRHL2 mRNA and protein expression in cisplatin-resistant ovarian serous papillary cystadenocarcinoma cell line (SKOV3/DDP) and the parental cell line (SKOV3). GRHL2 mRNA and protein expression in SKOV3/DDP was significantly higher compared to SKOV3 (Figure 1E-G).

\section{Establishment of Stable GRHL2} Knockdown and Overexpression Cell Lines

To further validate the purpose of GRHL2 in cisplatin resistance of SOC, we constructed GRHL2 stable knockdown SKOV3/DDP cells (respectively named SKOV3/DDPshGRHL2-1, SKOV3/DDP- shGRHL2-2, SKOV3/DDPshGRHL2-3) and the control cell (named SKOV3/DDPshcontrol). Meanwhile, pLV-EGFP vector contained the full length of GRHL2 sequence was transfected in SKOV3 cells (named SKOV3-GRHL2). The Empty pLV-EGFP vector was functioned as a negative control (named SKOV3-control). The knockdown and overexpression efficiency of GRHL2 was verified by qRT-PCR and Western blot (Figure 2). SKOV3/ DDP-shGRHL2-3 was selected as stable GRHL2 knockdown cell line for subsequent experiments.

\section{GRHL2 Expression Promotes Cisplatin- Resistance in vitro}

Next, we sought to validate the role of GRHL2 in cisplatin resistance of ovarian serous papillary cystadenocarcinoma cells. CCK-8 assay was conducted to assess the effect of GRHL2 knockdown and overexpression on the $\mathrm{IC}_{50}$ value of DDP. GRHL2 knockdown significantly attenuated cell viability, while GRHL2 overexpression did the opposite (Figure 3A and $\mathrm{B}$ ). The $\mathrm{IC}_{50}$ for DDP in SKOV3/DDP-shGRHL2 cells was appreciably lower compared to SKOV3/DDP-shcontrol cells (29.714 vs. $126.052 \mu \mathrm{g} / \mathrm{mL} ; P<0.05)$ (Figure 3C). In contrast, the $\mathrm{IC}_{50}$ value for DDP in SKOV3-GRHL2 cells was significantly increased compared to SKOV3-control cells (65.092 vs. $19.421 \mu \mathrm{g} / \mathrm{mL} ; P<0.05)$ (Figure 3D).

\section{GRHL2 Expression Promotes \\ Proliferation in vitro}

CCK-8 assays were conducted to determine the influence of GRHL2 on cell proliferation. A significant reduced growth rate was seen in SKOV3/DDP-shGRHL2 cells in relation to SKOV3/DDP-shcontrol cells, while GRHL2 overexpression resulted in a significant increased proliferation rate in SKOV3-GRHL2 cells relative to SKOV3control cells (Figure 3E and F).

\section{GRHL2 Expression Retards Cell Apoptosis in vitro}

Annexin V-APC/PI based FCM analysis was conducted to assess the influence of GRHL2 on cell apoptosis. A 
Table 2 Univariate and Multivariate Analysis for the Association Between Clinical Characteristics and Progression-Free Survival in Patients with SOC

\begin{tabular}{|c|c|c|c|c|}
\hline \multirow[t]{2}{*}{ Variable } & \multirow{2}{*}{$\begin{array}{l}\text { Univariate } \\
\text { HR (95\% Cl) }\end{array}$} & \multirow[t]{2}{*}{$P$ value } & Multivariate & \multirow[t]{2}{*}{$P$ value } \\
\hline & & & HR (95\% CI) & \\
\hline \multicolumn{5}{|l|}{ Age (year) } \\
\hline$<50$ & 1 & & & \\
\hline$\geq 50$ & $1.403(0.892-2.207)$ & 0.143 & & \\
\hline \multicolumn{5}{|l|}{ FIGO stage } \\
\hline I-II & 1 & & I & \\
\hline III-IV & $2.410(1.425-4.077)$ & 0.001 & $1.864(1.062-3.269)$ & 0.030 \\
\hline \multicolumn{5}{|l|}{ Histological grade } \\
\hline Low & 1 & & & \\
\hline High & $1.221(0.733-2.034)$ & 0.443 & & \\
\hline \multicolumn{5}{|l|}{ Lymph node invasion } \\
\hline Negative & 1 & & I & \\
\hline Positive & $2.133(1.221-3.726)$ & 0.008 & $1.016(0.428-2.410)$ & 0.972 \\
\hline \multicolumn{5}{|l|}{ Tumor residual(cm) } \\
\hline Optimal $(\leq 1)$ & I & & I & \\
\hline Sub-optimal(>I) & $2.277(1.305-3.974)$ & 0.004 & $1.074(0.456-2.53 \mathrm{I})$ & 0.870 \\
\hline \multicolumn{5}{|l|}{ GRHL2 expression } \\
\hline Low & 1 & & 1 & \\
\hline High & $4.040(2.388-6.835)$ & $<0.001$ & $3.448(1.950-6.097)$ & $<0.001$ \\
\hline
\end{tabular}

Note: The bold number represents the $P$-values with significant differences $(P<0.05)$.

Abbreviations: $\mathrm{HR}$, hazard ratio; $\mathrm{Cl}$, confidence intervals.

significant higher apoptosis rate was seen in SKOV3/ DDP-shGRHL2 cells in relation to SKOV3/DDP-shcontrol cells (Figure 3G and $\mathrm{H}$ ), while GRHL2 overexpression resulted in a significant lower apoptosis rate in SKOV3GRHL2 cells in relation to SKOV3-control cells (Figure 3I and J).

\section{GRHL2 Expression Activates ERK/MAPK Signaling Pathways in vitro}

Previous studies have reported that the ERK/MAPK signaling pathways mediate chemoresistance with various mechanisms, including increased cell proliferation, and reduced cell apoptosis. ${ }^{29-33}$ To discover the molecular mechanism of GRHL2-regulated chemoresistance in SOC, we conducted Western blot assays to confirm the effects of GRHL2 on ERK/MAPK signaling pathways. Results indicated that knockdown of GRHL2 led to a significant decrease in expression of Ki-67, Cyclin D1, p-ERK proteins and higher expression of Fas, caspase 3 proteins, while ERK protein expression showed inconspicuous variations (Figure 4A and B). Next, we utilized
Western blot assays to identify variations associated with GRHL2 overexpression. Importantly, treatment with GRHL2 overexpression plus ERK/MAPK kinase inhibitor (U0126, $10.0 \mu \mathrm{M}$ ) could rescue the changes associated with GRHL2 overexpression (Figure 4C and D). These results indicated that GRHL2 promoted DDP resistance in SOC cells by increasing cell proliferation and reducing cell apoptosis via stimulation of ERK/MAPK signaling pathways.

\section{GRHL2 Expression Increases Cisplatin- Resistance in vivo}

In addition, we assessed the consequence of GRHL2 knockdown or overexpression on the sensitivity of cisplatin-tolerant cells and its parental cell to DDP in nude mice. Chemotherapy regimens were initiated once the average tumor size extended to approximately $50 \mathrm{~mm}^{3}$ (Day12). DDP was administered using intraperitoneal injection at a dose of $3.0 \mathrm{mg} / \mathrm{kg}$ six times with a gap of 3 days. As shown in Figure 5A and B, GRHL2 knockdown evidently suppressed tumor growth. After DDP therapy, a significantly 
Table 3 Univariate and Multivariate Analysis for the Association Between Clinical Characteristics and Overall Survival in Patients with SOC

\begin{tabular}{|c|c|c|c|c|}
\hline \multirow[t]{2}{*}{ Variable } & \multirow{2}{*}{$\begin{array}{l}\text { Univariate } \\
\text { HR (95\% Cl) }\end{array}$} & \multirow[t]{2}{*}{$P$ value } & Multivariate & \multirow[t]{2}{*}{$P$ value } \\
\hline & & & HR (95\% Cl) & \\
\hline \multicolumn{5}{|l|}{ Age(year) } \\
\hline$<50$ & I & & I & \\
\hline$\geq 50$ & 1.953 (1.189-3.209) & 0.008 & $1.966(1.117-3.460)$ & 0.019 \\
\hline \multicolumn{5}{|l|}{ FIGO stage } \\
\hline I-II & 1 & & I & \\
\hline III-IV & 6.691 (3.155-14.192) & $<0.001$ & $5.297(2.256-12.437)$ & $<0.001$ \\
\hline \multicolumn{5}{|l|}{ Histological grade } \\
\hline Low & I & & I & \\
\hline High & $1.922(1.091-3.389)$ & 0.024 & $0.904(0.456-1.794)$ & 0.773 \\
\hline \multicolumn{5}{|l|}{ Lymph node invasion } \\
\hline Negative & I & & I & \\
\hline Positive & $3.576(1.994-6.412)$ & $<0.001$ & $0.647(0.238-1.762)$ & 0.395 \\
\hline \multicolumn{5}{|l|}{ Tumor residual $(\mathrm{cm})$} \\
\hline Optimal $(\leq 1)$ & I & & I & \\
\hline Sub-optimal(>I) & $3.967(2.213-7.113)$ & $<0.001$ & $2.092(0.802-5.458)$ & 0.131 \\
\hline \multicolumn{5}{|l|}{ GRHL2 expression } \\
\hline Low & I & & I & \\
\hline High & $5.717(3.202-10.207)$ & $<0.001$ & $4.880(2.559-9.308)$ & $<0.001$ \\
\hline
\end{tabular}

Note: The bold number represents the $P$-values with significant differences $(P<0.05)$.

Abbreviations: $\mathrm{HR}$, hazard ratio; $\mathrm{Cl}$, confidence intervals.

lower growth rate was observed in tumors formed SKOV3/ DDP-shGRHL2 cells relative to SKOV3/DDP-shcontrol cells. In contrast, tumors formed by SKOV3/GRHL2 cells were considerably bigger than tumors developed by SKOV3control cells. After DDP therapy, a significantly faster growth rate was observed in tumors formed by SKOV3-GRHL2 cells relative to SKOV3-control cells (Figure 5C and D). Therefore, GRHL2 encouraged resistance of serous ovarian cancer cells to DDP in vivo.

\section{Discussion}

GRHL2, a transcriptional regulator of proliferation and differentiation of epithelial cells, has been validated to play a vital function in various life processes. The relevance of GRHL2 in cancer occurrence and development varies and can play different roles as both an oncogene as well as tumor suppressor depending on the tumor type. Recently, GRHL2 has been reported to be upregulated in epithelial ovarian cancer (EOC) and encouraged the proliferation, migration and invasive ability of EOC cells. ${ }^{25}$ Nevertheless, the association between the GRHL2 expression and sensitivity of SOC to cisplatin is not clear and needs to be additionally clarified. Interestingly, GRHL2 was identified to be a hypomethylated gene in high grade serous ovarian cancer. ${ }^{34}$ It is known that differential DNA methylation contributes to platinum-resistance of SOC. ${ }^{35}$ Also, Dompe et al found that GRHL2 can inhibit death receptor-mediated apoptosis by down-regulating the expression of the death receptor Fas and DR5 in breast cancer. Therefore, studies have suggested that down-regulation of GRHL2 expression and functional silencing can be used as clinical treatment strategy to improve the response of death receptors to drugs and play a role in tumor therapy. ${ }^{36}$ Hence, we speculated that GRHL2 may play a role in chemoresistance of SOC.

In this study, we first performed immunohistochemistry to identify GRHL2 protein expression in SOC tissues. We determined that there was higher expression of GRHL2 in DDP resistant SOC tissues relative to DDP sensitive SOC tissues. In addition, high expression of GRHL2 in SOC tissues led to short PFS and OS of SOC patients. Secondly, we identified GRHL2 mRNA and protein expression in DDP resistant ovarian serous papillary cystadenocarcinoma cell line SKOV3/DDP and its parent cell line 

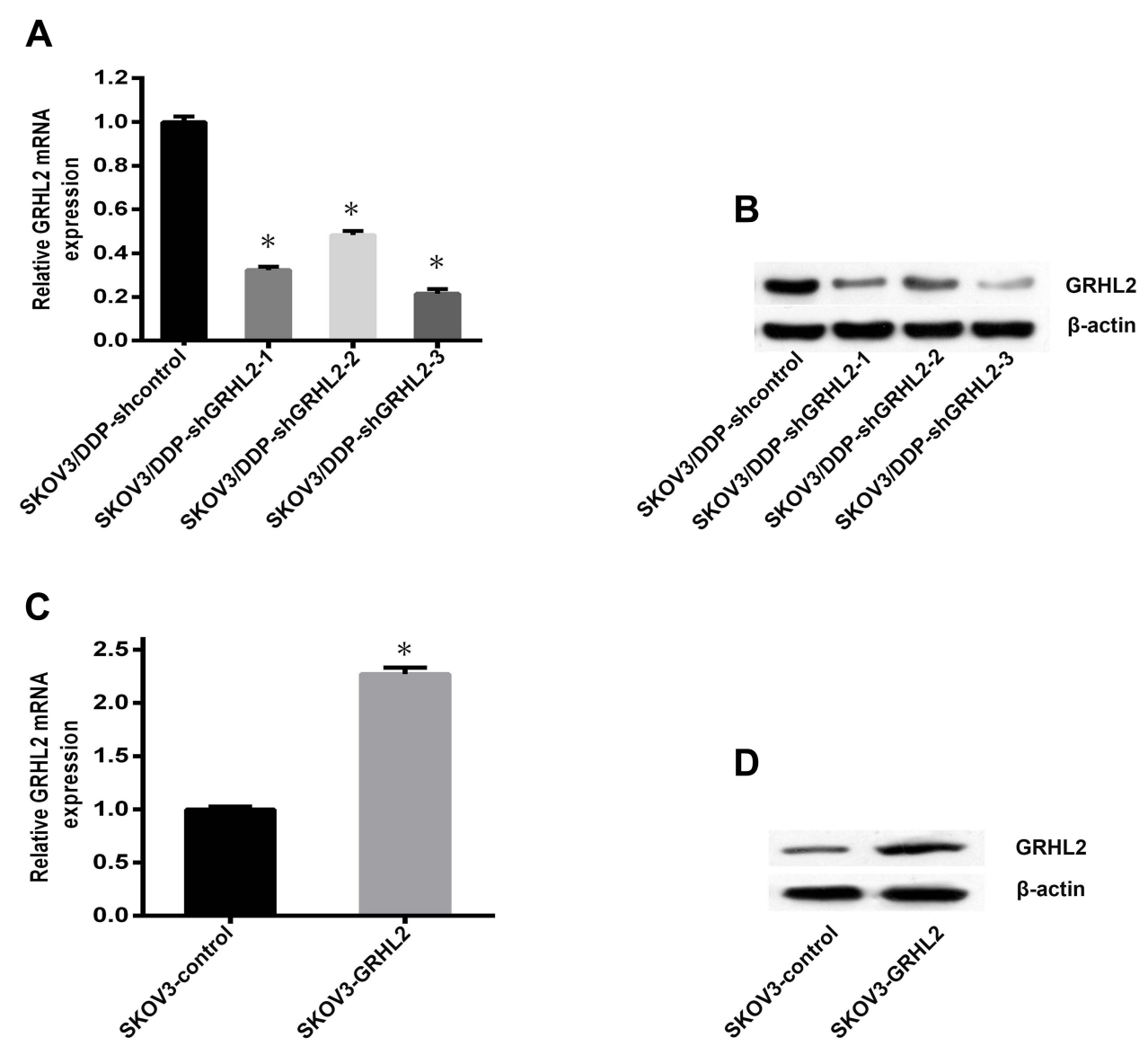

Figure 2 The knockdown and overexpression efficiency of GRHL2 were detected by qRT-PCR and Western blot. (A, B) Both the relative mRNA and protein levels of GRHL2 were deeply suppressed by GRHL2 knockdown. $* P<0.05$ vs SKOV3/DDP-shcontrol. (C, D) Both the relative mRNA and protein levels of GRHL2 were deeply promoted by GRHL2 overexpression. $* P<0.05$ vs SKOV3-control.

Notes: Data were expressed as mean $\pm S D$.

Abbreviation: $q R T-P C R$, quantitative real-time PCR.

(SKOV3). It was found that GRHL2 mRNA and protein expression in SKOV3/DDP cell line were considerably increased compared to the SKOV3 cell line. This data suggests that the up-regulation of GRHL2 may regulate resistance of SOC to DDP, which affects the prognosis of SOC patients.

To prove this, we conducted an analysis of gain- and loss-of-function. Firstly, we used RNA interference technique to down-regulate the endogenous GRHL2 in SKOV3/DDP cells, and demonstrated that GRHL2 knockdown heightens the sensitivity of SKOV3/DDP cells to DDP in vitro and in vivo by inhibiting proliferation and promoting apoptosis. Secondly, we used gene overexpression technique to up-regulate GRHL2 in SKOV3 cells. The results showed that the up-regulation of GRHL2 could promote proliferation and inhibit apoptosis, which could lead to the decrease of sensitivity of SKOV3 cells to
DDP in vitro and in vivo. Subsequently, we studied the potential molecular mechanism of GRHL2-mediated resistance of ovarian cancer cells to DDP.

It has been reported that GRHL2 knockout in SKOV3 cells leads to a significant down-regulation of many standard carcinogenic pathways related to cell growth and proliferation, of which the down-regulation of ERK/ MAPK is the most significant. ${ }^{25}$ Interestingly, previous studies have reported that the ERK/MAPK signaling pathways mediate chemoresistance with various mechanisms, including increased cell proliferation and reduced cell apoptosis. In breast, ${ }^{29}$ gastric, ${ }^{30}$ colon and endometrial cancers, ${ }^{31,32}$ ERK1/2 induces a multi-drug resistant phenotype by increasing anti-apoptotic proteins belonging to the Bcl-2 family. Moreover, increased cell growth and decreased apoptosis induced by ERK1/2 also causes resistance to various chemotherapeutic drugs in ovarian 

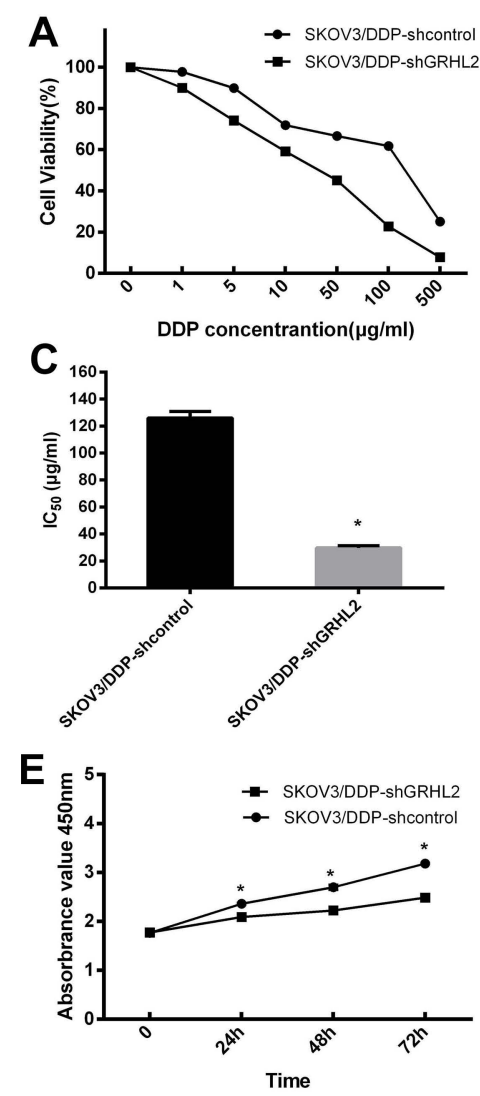

G
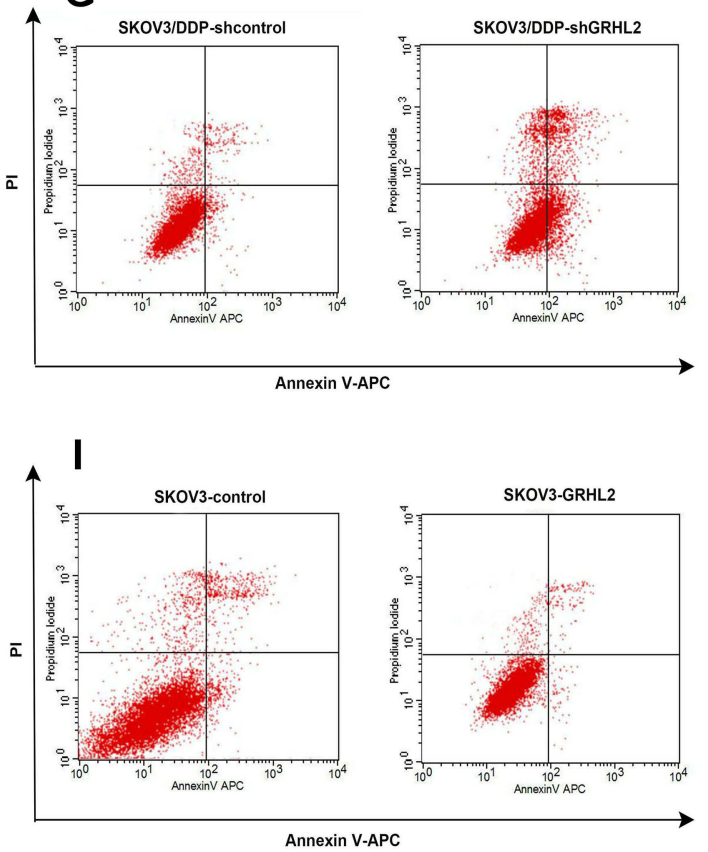
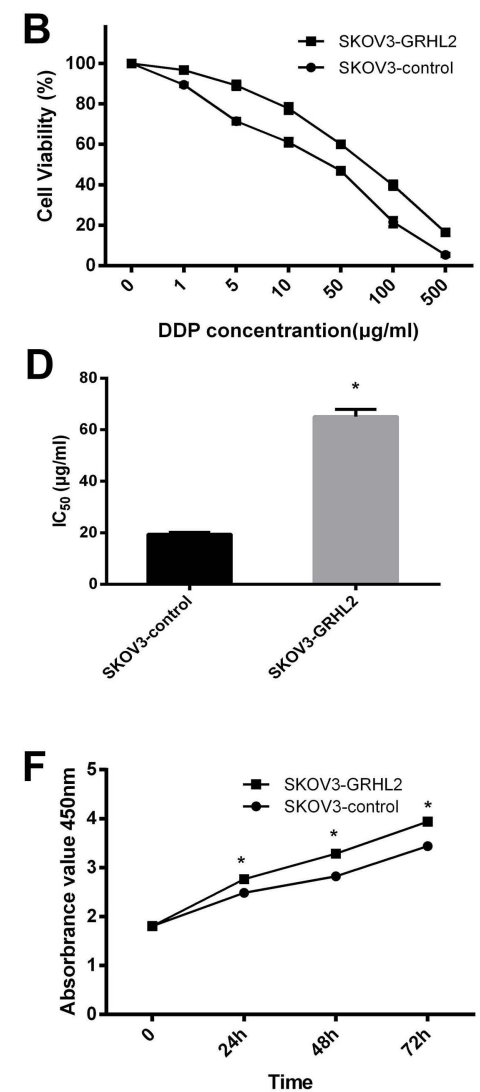

H

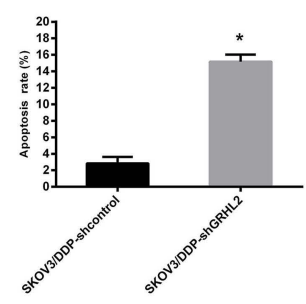

$\mathbf{J}$

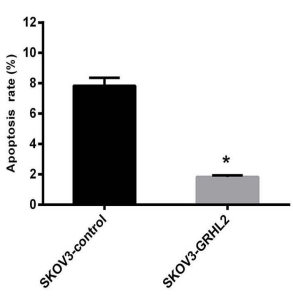

Figure 3 GRHL2 increased cisplatin-resistance, promoted cell proliferation and retarded cell apoptosis in vitro. (A, C) CCK-8 analysis was used to determine the cell viability and the $I_{50}$ values of DDP in SKOV3/DDP-shGRHL2 and SKOV3/DDP-shcontrol cells. $* P<0.05$ vs SKOV3/DDP-shcontrol. (B, D) CCK-8 analysis was used to evaluate the cell viability and the $\mathrm{IC}_{50}$ values of DDP in SKOV3-GRHL2 and SKOV3-control cells. $* P<0.05$ vs SKOV3-control. (E) CCK-8 assays were performed to determine the effects of GRHL2 knockdown on the proliferation of SKOV3/DDP cells. $* P<0.05$ vs SKOV3/DDP-shcontrol. (F) CCK-8 assays were performed to determine the effects of GRHL2 upregulation on the proliferation of SKOV3 cells. *P<0.05 vs SKOV3-control. (G, H) Apoptosis of SKOV3/DDP-shGRHL2 and SKOV3/DDP-shcontrol cells was evaluated by FCM. $* P<0.05$ vs SKOV3/DDP-shcontrol. (I, J) Apoptosis of SKOV3-GRHL2 and SKOV3-control cells by FCM. $* P<0.05$ vs SKOV3-control.

Note: Data were expressed as mean \pm SD.

Abbreviations: CCK-8, Cell Counting Kit-8; IC 50 , half-maximal inhibitory concentration. 
A

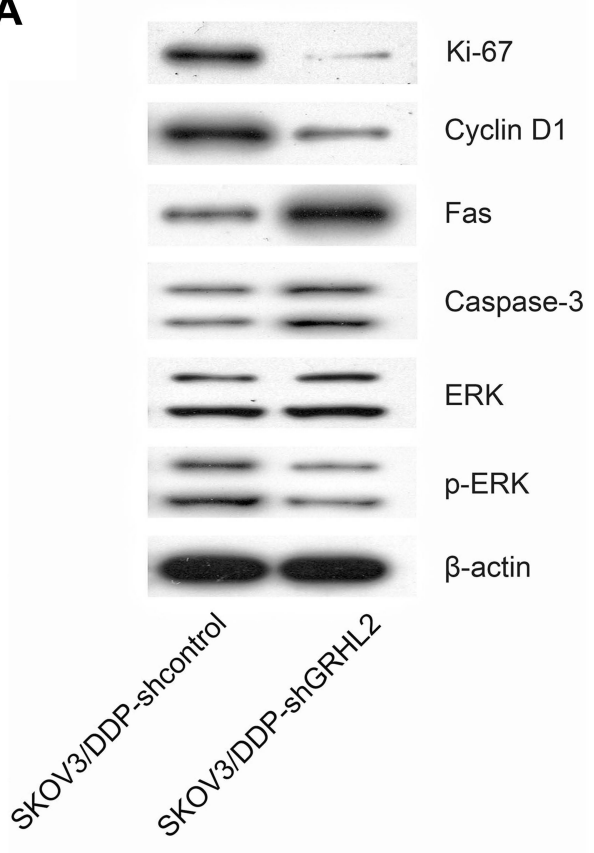

B

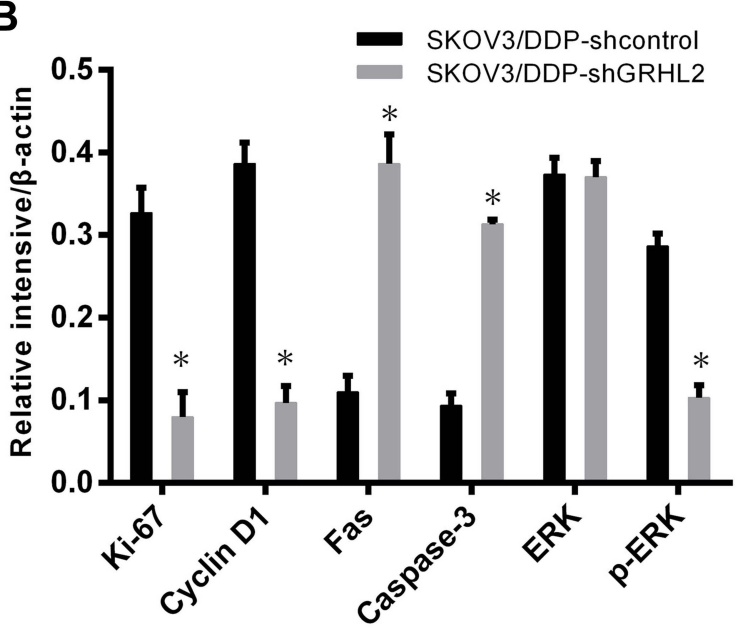

C
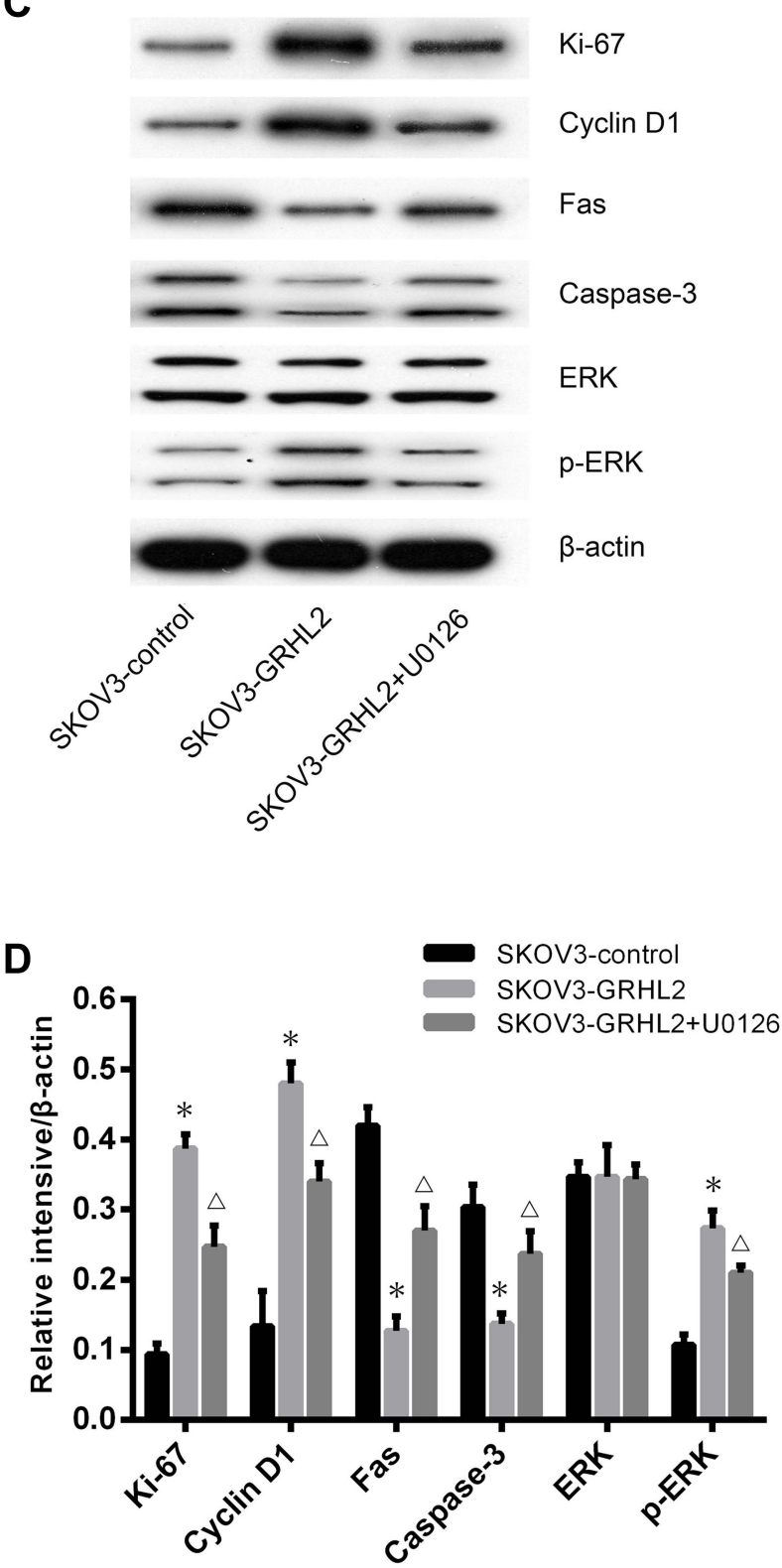

Figure 4 GRHL2 expression activated ERK/MAPK signaling pathways in vitro. (A, B) Western blot was used to detect the expression of Fas, caspase3, Ki-67, Cyclin DI, P-ERK and total ERK proteins in SKOV3/DDP-shGRHL2 or SKOV3/DDP-shcontrol cells, respectively. ${ }^{* P}<0.05$ vs SKOV3/DDP-shcontrol. (C, D) Western blot was used to evaluate the expression of Fas, caspase3, Ki-67, Cyclin DI, p-ERK and total ERK proteins in SKOV3-GRHL2, SKOV3-control cells and SKOV3-GRHL2 cells treated with U0I $26($ I $0.0 \mu \mathrm{M})$, respectively. ${ }^{*} P<0.05$ vs SKOV3-control; $\triangle P<0.05$ vs SKOV3-GRHL2.

Note: Data were expressed as mean \pm SD.

cancer. $^{33}$ However, it is not clear whether GRHL2 encourages resistance of ovarian cancer cells to DDP by activating the ERK/MAPK signaling pathway. We found that GRHL2 knockdown could reduce the expression of Ki-67, Cyclin D1, p-ERK proteins in SKOV3/DDP cells. However, expression of total ERK protein did not change, and then Fas, caspase 3 proteins increased. At the same time, up-regulation of GRHL2 can lead to the increase of $\mathrm{Ki}-67$, Cyclin D1, p-ERK proteins expression in SKOV3 cells, which in turn led to the decrease of Fas and caspase3. Importantly, treatment with ERK/MAPK kinase inhibitors could reverse the changes of these protein in SKOV3/GRHL2 cells induced by GRHL2 up-regulation. Therefore, the regulation of Ki-67, Cyclin D1, Fas and caspase-3 by GRHL2 may be the result of activating the ERK/MAPK signaling pathway. However, the possible molecular mechanism of ERK/MAPK pathway activation mediated by GRHL2 needs to be further studied. 
A

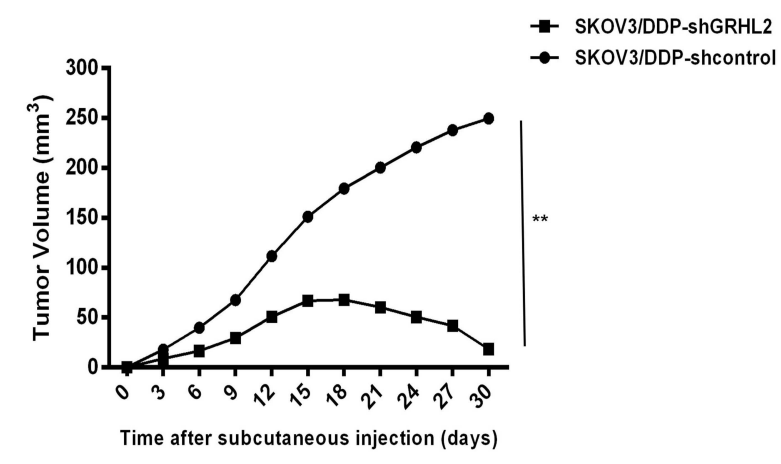

B

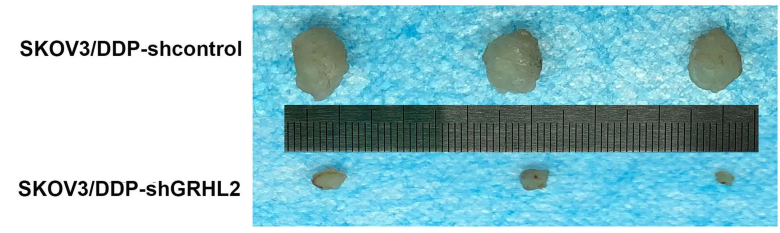

C

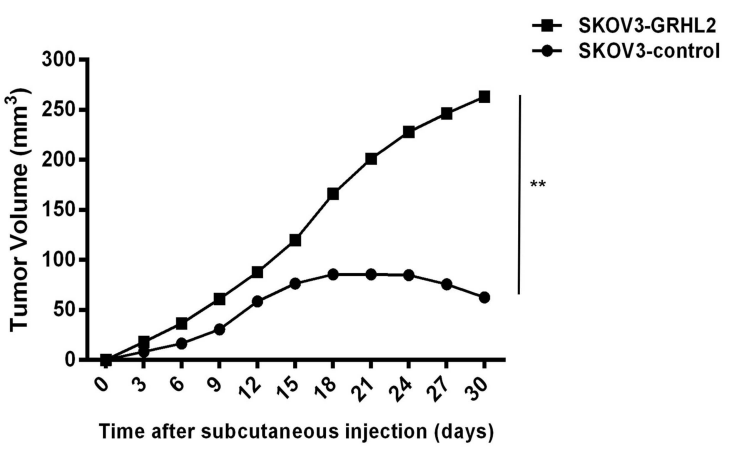

D

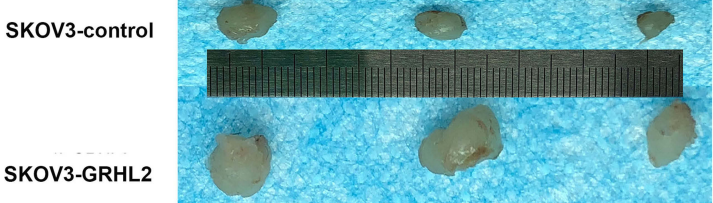

Figure 5 GRHL2 expression increased cisplatin-resistance in vivo. (A, B) The growth in mice of tumors developed from SKOV3/DDP-shGRHL2 or SKOV3/DDP-shcontrol cells were treated with cisplatin $\left(3.0 \mathrm{mg} / \mathrm{kg}\right.$ six times with a gap of 3 days). ${ }^{* *} ; P<0.01$ vs SKOV3/DDP -shcontrol. (C, D) The growth in mice of tumors developed from SKOV3-GRHL2 or SKOV3-control cells were treated with cisplatin $(3.0 \mathrm{mg} / \mathrm{kg}$ six times with a gap of 3 days $)$. $* * P<0.01$ vs SKOV3-control cells. Note: $n=3$ per group.

\section{Conclusions}

GRHL2 was significantly up-regulated in DDP-resistant ovarian cancer tissues and cell lines. GRHL2 encouraged cell proliferation and reduced apoptosis by activating ERK/MAPK signaling pathway, thus promoting the resistance of ovarian cancer cells to DDP. Therefore, GRHL2 may represent a possible treatment target for cisplatinresistant serous ovarian cancer.

\section{Ethics Approval and Informed Consent}

The Ethics Committee of Second Xiangya Hospital of CSU granted approval to the study. All participants signed informed consent forms. The Institutional Animal Care and Use Committee of Second Xiangya Hospital of CSU granted approval to the animal study. All experiments were in accordance with the 1964 Helsinki declaration and with the guidelines of the Institutional Animal Care and Use Committee of Second Xiangya Hospital of CSU.

\section{Disclosure}

The authors report no conflicts of interest in this work.

\section{References}

1. Jayson GC, Kohn EC, Kitchener HC, Ledermann JA. Ovarian cancer. Lancet (London, England). 2014;384(9951):1376-1388. doi:10.1016/ S0140-6736(13)62146-7

2. Torre LA, Trabert B, DeSantis CE, et al. Ovarian cancer statistics, 2018 CA Cancer J Clin. 2018;68(4):284-296. doi:10.3322/caac.21456

3. Siegel RL, Miller KD, Jemal A. Cancer statistics, 2019. CA Cancer J Clin. 2019;69(1):7-34. doi:10.3322/caac.21551

4. Ledermann JA, Raja FA, Fotopoulou C, Gonzalez-Martin A, Colombo $\mathrm{N}$, Sessa C. Newly diagnosed and relapsed epithelial ovarian carcinoma: ESMO clinical practice guidelines for diagnosis, treatment and followup. Ann Oncol. 2018;29(Suppl 4):iv259. doi:10.1093/annonc/mdy157

5. McQuade RM, Stojanovska V, Bornstein JC, Nurgali K. PARP inhibition in platinum-based chemotherapy: chemopotentiation and neuroprotection. Pharmacol Res. 2018;137:104-113. doi:10.1016/j.phrs.2018.09.031

6. Taylor SE, Petschauer JS, Donovan H, et al. Phase I study of intravenous oxaliplatin and intraperitoneal docetaxel in recurrent ovarian cancer. Int J Gynecol Cancer. 2019;29(1):147-152. doi:10.1136/ijgc2018-000055

7. van Zyl B, Tang D, Bowden NA. Biomarkers of platinum resistance in ovarian cancer: what can we use to improve treatment. Endocr Relat Cancer. 2018;25(5):R303-R318. doi:10.1530/ERC-17-0336

8. Pyrgaki C, Liu A, Niswander L. Grainyhead-like 2 regulates neural tube closure and adhesion molecule expression during neural fold fusion. Dev Biol. 2011;353(1):38-49. doi:10.1016/j.ydbio.2011.02.027

9. Chen W, Xiao Liu Z, Oh JE, et al. Grainyhead-like 2 (GRHL2) inhibits keratinocyte differentiation through epigenetic mechanism. Cell Death Dis. 2012;3:e450. doi:10.1038/cddis.2012.190

10. Walentin K, Hinze C, Werth M, et al. A Grhl2-dependent gene network controls trophoblast branching morphogenesis. Development (Cambridge, England). 2015;142(6):1125-1136. doi:10.1242/dev.11 3829 
11. Senga K, Mostov KE, Mitaka T, Miyajima A, Tanimizu N, Nusrat A. Grainyhead-like 2 regulates epithelial morphogenesis by establishing functional tight junctions through the organization of a molecular network among claudin3, claudin4, and Rab25. Mol Biol Cell. 2012;23(15):2845-2855. doi:10.1091/mbc.e12-02-0097

12. Tanimizu N, Nakamura $\mathrm{Y}$, Ichinohe N, Mizuguchi T, Hirata K, Mitaka T. Hepatic biliary epithelial cells acquire epithelial integrity but lose plasticity to differentiate into hepatocytes in vitro during development. J Cell Sci. 2013;126(Pt 22):5239-5246. doi:10.1242/ jcs. 133082

13. Van Laer L, Van Eyken E, Fransen E, et al. The grainyhead like 2 gene (GRHL2), alias TFCP2L3, is associated with age-related hearing impairment. Hum Mol Genet. 2008;17(2):159-169. doi:10.1093/ hmg/ddm292

14. Chi D, Singhal H, Li L, et al. Estrogen receptor signaling is reprogrammed during breast tumorigenesis. Proc Natl Acad Sci U S A. 2019;116(23):11437-11443. doi:10.1073/pnas.1819155116

15. Holding AN, Giorgi FM, Donnelly A, et al. VULCAN integrates ChIP-seq with patient-derived co-expression networks to identify GRHL2 as a key co-regulator of ERa at enhancers in breast cancer. Genome Biol. 2019;20(1):91. doi:10.1186/s13059-019-1698-z

16. Quan Y, Xu M, Cui P, Ye M, Zhuang B, Min Z. Grainyhead-like 2 promotes tumor growth and is associated with poor prognosis in colorectal cancer. $J$ Cancer. 2015;6(4):342-350. doi:10.7150/ jca.10969

17. Yang Z, Wu D, Chen Y, Min Z, Quan Y. GRHL2 inhibits colorectal cancer progression and metastasis via oppressing epithelial-mesenchymal transition. Cancer Biol Ther. 2019;20(9):1195-1205. doi:10.1080/15384047.2019.1599664

18. Hu F, He Z, Sun C, Rong D. Knockdown of GRHL2 inhibited proliferation and induced apoptosis of colorectal cancer by suppressing the PI3K/Akt pathway. Gene. 2019;700:96-104. doi:10.1016/j. gene.2019.03.051

19. Xiang J, Fu X, Ran W, Wang Z. Grhl2 reduces invasion and migration through inhibition of TGFbeta-induced EMT in gastric cancer. Oncogenesis. 2017;6(1):e284. doi:10.1038/oncsis.2016.83

20. Nishino H, Takano S, Yoshitomi H, et al. Grainyhead-like 2 (GRHL2) regulates epithelial plasticity in pancreatic cancer progression. Cancer Med. 2017;6(11):2686-2696. doi:10.1002/cam4.1212

21. Chen W, Yi JK, Shimane T, et al. Grainyhead-like 2 regulates epithelial plasticity and stemness in oral cancer cells. Carcinogenesis. 2016;37(5):500-510. doi:10.1093/carcin/bgw027

22. Paltoglou S, Das R, Townley SL, et al. Novel androgen receptor coregulator GRHL2 exerts both oncogenic and antimetastatic functions in prostate cancer. Cancer Res. 2017;77(13):3417-3430. doi:10.1158/0008-5472.CAN-16-1616

23. Pistore C, Giannoni E, Colangelo T, et al. DNA methylation variations are required for epithelial-to-mesenchymal transition induced by cancer-associated fibroblasts in prostate cancer cells. Oncogene. 2017;36(40):5551-5566. doi:10.1038/onc.2017.159
24. Chung VY, Tan TZ, Tan M, et al. GRHL2-miR-200-ZEB1 maintains the epithelial status of ovarian cancer through transcriptional regulation and histone modification. Sci Rep. 2016;6(1):19943. doi:10.1038/srep19943

25. Faddaoui A, Sheta R, Bachvarova M, et al. Suppression of the grainyhead transcription factor 2 gene (GRHL2) inhibits the proliferation, migration, invasion and mediates cell cycle arrest of ovarian cancer cells. Cell Cycle. 2017;16(7):693-706. doi:10.1080/ 15384101.2017.1295181

26. Therasse P, Arbuck SG, Eisenhauer EA, et al. New guidelines to evaluate the response to treatment in solid tumors. European Organization for Research and Treatment of Cancer, National Cancer Institute of the United States, National Cancer Institute of Canada. J Natl Cancer Inst. 2000;92(3):205-216. doi:10.1093/jnci/92.3.205

27. Berek JS, Kehoe ST, Kumar L, Friedlander M. Cancer of the ovary, fallopian tube, and peritoneum. Int J Gynaecol Obstet. 2018;143 (Suppl 2):59-78. doi:10.1002/ijgo.12614

28. Shimizu M, Saitoh Y, Itoh H. Immunohistochemical staining of Haras oncogene product in normal, benign, and malignant human pancreatic tissues. Hum Pathol. 1990;21(6):607-612. doi:10.1016/ S0046-8177(96)90006-4

29. Chen D, Si W, Shen J, et al. miR-27b-3p inhibits proliferation and potentially reverses multi-chemoresistance by targeting CBLB/GRB2 in breast cancer cells. Cell Death Dis. 2018;9(2):188. doi:10.1038/ s41419-017-0211-4

30. Jiang X, Jiang X, Yang Z. NRAGE confers poor prognosis and promotes proliferation, invasion, and chemoresistance in gastric cancer. Gene. 2018;668:114-120. doi:10.1016/j.gene.2018.05.060

31. Li S, Tian J, Zhang H, et al. Down-regulating IL-6/GP130 targets improved the anti-tumor effects of 5-fluorouracil in colon cancer. Apoptosis. 2018;23(5-6):356-374. doi:10.1007/s10495-018-1460-0

32. Guo F, Zhang H, Jia Z, Cui M, Tian J. Chemoresistance and targeting of growth factors/cytokines signalling pathways: towards the development of effective therapeutic strategy for endometrial cancer. $\mathrm{Am} J$ Cancer Res. 2018;8(7):1317-1331.

33. Liu S, Zha J, Lei M. Inhibiting ERK/Mnk/eIF4E broadly sensitizes ovarian cancer response to chemotherapy. Clin Transl Oncol. 2018;20 (3):374-381. doi:10.1007/s12094-017-1724-0

34. Keita M, Wang ZQ, Pelletier JF, et al. Global methylation profiling in serous ovarian cancer is indicative for distinct aberrant DNA methylation signatures associated with tumor aggressiveness and disease progression. Gynecol Oncol. 2013;128(2):356-363. doi:10.1016/j. ygyno.2012.11.036

35. Borley J, Brown R. Epigenetic mechanisms and therapeutic targets of chemotherapy resistance in epithelial ovarian cancer. Ann Med. 2015;47(5):359-369. doi:10.3109/07853890.2015.1043140

36. Dompe N, Rivers CS, Li L, et al. A whole-genome RNAi screen identifies an 8q22 gene cluster that inhibits death receptor-mediated apoptosis. Proc Natl Acad Sci U S A. 2011;108(43):E943-E951. doi:10.1073/pnas.1100132108
OncoTargets and Therapy

\section{Publish your work in this journal}

OncoTargets and Therapy is an international, peer-reviewed, open access journal focusing on the pathological basis of all cancers, potential targets for therapy and treatment protocols employed to improve the management of cancer patients. The journal also focuses on the impact of management programs and new therapeutic agents and protocols on patient perspectives such as quality of life, adherence and satisfaction. The manuscript management system is completely online and includes a very quick and fair peer-review system, which is all easy to use. Visit http://www.dovepress.com/ testimonials.php to read real quotes from published authors. 